\title{
Mimo System using16 Psk and Bpsk Modulation Schemes
}

\section{G. Navabharat Reddy, D. Kiran Kumar, Sruti Setlam, Harshitha S}

\begin{abstract}
Current technology will build on signals mutual independent recurrence Technique(Orthogonal frequency divison multiplexing) and we don't know the quality of it altogether expanded in numerous inputs numerous yields like Multi Input Multi output, Taking a shot at the same objective we use the LDPC based MIMO-OFDM with 16PSK and Binary modulation schemes. The bit error rate is checked so that we identify the which scheme is best.
\end{abstract}

Keywords: MIMO,OFDM, LDPC, BER.

\section{INTRODUCTION}

Information is transmitted in different ways. This is done in better way using the available resources with high performance. When the user tends to move at the higher speed, the need to send the data through the different media has become a challenging for the past few years.

Since the world is digitalized, everyone are more depends on electronic gadgets most of the time. This leads to increase the demand in speed so we are going to develop different communication techniques. To overcome these demands, we need to adopt some new technologies. Namely, Orthogonal Frequency Division Multiplexing (OFDM) is proposed. It uses different carriers to technique. Here the information goes in a different way. The main advantage is signals are independent so that no overlap of signals. The low-density parity-check is identify the errors in data. Here the information's gets transmitted over a noisy channels. The technique which allows work based on Pilot aied Transmission (PAT). In the recent years, these types of technologies are most widely used in many of the new generation communication systems.

Nowadays, without wire devices i.e electronic devices available everywhere. Thus, the need for the bandwidth efficient communication systems has been increased widely and become the major demand. Shannon limit has delivered to all users and speed is maintain according to limitation of channel bandwith. In case, when the power is not constrained then there exist an infinite capacity.

The fundamental idea of this technique is carriers are independent of each other so this is very good result oriented techinque. This method work on signal

Revised Manuscript Received on April 25, 2020.

* Correspondence Author

G. Navabharat Reddy*, Assistant Professor, ECE, Vignan Institute of Technolgoy and science

D. Kiran Kumar, Assistant professor, ECE, Vignan institute of Technology and science

Sruthi Setlam, Assistant professor ECE.

Harshitha S, Assistant professor ECE, Siddhartha Institute of engineering Techonlogy

(C) The Authors. Published by Blue Eyes Intelligence Engineering and Sciences Publication (BEIESP). This is an open access article under the CC BY-NC-ND license (http://creativecommons.org/licenses/by-nc-nd/4.0/) independency so that it match speed so it will maintained frequency. So there is no interference in this method so data is free from noise so most of the time we prefer this method.

With a specific end goal the main of this method is no noise and signals are mutually exclusive to each other in that way we maintain carriers. The Orthogonal frequency divison multiplexing framework transmits countless transporters, which are firmly dispersed. So most of the time this technique applied in data transmission methods data can be move all the way without any external and internal disturbance that's why we also prefer this method after applying so much of research every method has there own benefits and losses this also there own set of rules .

\section{MIMO-OFDM:}

MIMO systems consist of multiple transmitters and receivers. The system capacity can be increased. In-case of MIMO, signals at different Transducer undergoes losses in a different weathers so devices are placed at a finite distance.

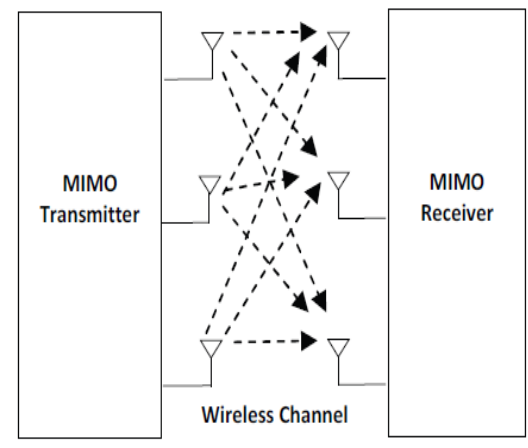

Fig. 1 MIMO Channel

Orthogonal Frequency Division Multiplexing (OFDM) is a method in which the digital data's are encoded using multiple carrier frequencies. It is used in wideband digital communication systems. It is known for its applications in digital television and audio broadcasting, DSL internet access, power line networks and 4G mobile communication.

OFDM comes under FDM scheme where, a larger number of closely spaced orthogonal subcarrier signals that are used to carry data on several parallel data schemes. Conventional modulation schemes such as Quadrature Amplitude modulation (QAM) or Phase shift keying (PSK) are used to modulate these subcarriers. This is done at low symbol rate.

The main advantage of OFDM over single carrier modulation scheme is it capability to operate with severe channel condition like attenuation of high frequencies, narrow band interference and frequency selective fading due to multi-path without complex equalization filters. OFDM can be seen as a system which

Published By:

Blue Eyes Intelligence Engineering \& Sciences Publication

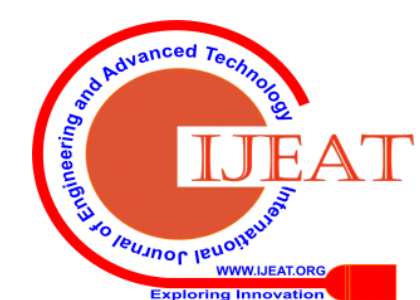




\section{Mimo System using16 Psk and Bpsk Modulation Schemes}

uses the slowly modulated narrow band signals when compared to one rapidly modulated wide band signal. Usage of Guard interval between the symbols of OFDM is possible because of low symbol rate and due to this Inter symbol Interference is eliminated. Utilization of echoes and time spreading helps to achieve a diversity gain that is improvement in signal to noise ratio.

\section{AWGN CHANNEL:}

Added substance white Gaussian clamor (AWGN) channel is a fundamental or usually utilized channel model for investigating modulation plans. In this model, the AWGN channel includes a white Gaussian clamor to the signal that passes through it. This suggests the channel's plentifulness recurrence reaction is level (hence with boundless or vast transmission capacity) and stage recurrence reaction is direct for all frequencies so that tweaked signals experience it with no abundance misfortune and stage contortion. Blurring does not exist for this channel. The transmitted sign gets bended just by AWGN process.

\section{WORKING PRINCIPLE:}

BPSK and 16PSK modulation is carried out on this paper. Initially the serial data input is formatted and transmitted in parallel form. Each data assigns one carrier to transmit then OFDM modulation is performed. Here the inverse Fourier transform is used. This converts signal from frequency domain to time domain and then the guard period is added to each symbol. There are two sections in guard period, first half has the zero time amplitude and the other hard is the extension of the cyclic prefix. Then additive white Gaussian voice is added to the channel from which snr is set. This process occurs at transmitter side. The reverse proposes is performed at receiver. Then the cyclic prefix is removed the fast Fourier transform is carried out to recover the original data. Finally the parallel data streams converted into serial form. This methodology is implemented in the MATLAB.

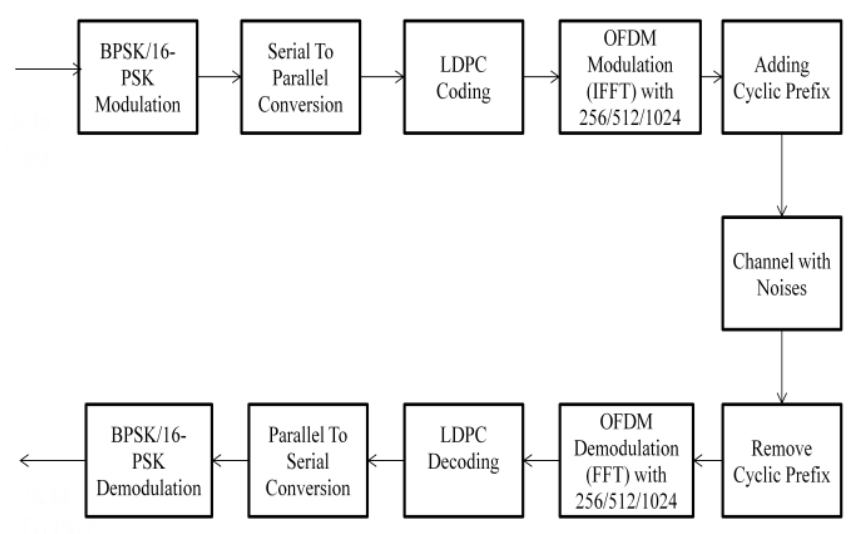

\section{ELEMENTS THAT AFFECTING BER}

There are various types of component which can impact Bit Error Rate are as per the following:

(A) Interference:

The obstruction levels present in a framework are by and large set by outer variables and can't be changed by the framework plan. Be that as it may it is conceivable to set the data transmission of the framework. By diminishing the data transmission the level of impedance can be diminished. However diminishing the transmission capacity confines the information throughput that can be accomplished

(B) Increase the force level:

It is possible to build the force level of the framework so that the force per bit is expanded. This has to be adjusted against component including the impedance levels to different clients and the effect of expanding the force yield on the measure of the force intensifier and general force utilization and battery life, and so forth.

\section{ANALYSIS OF THE BER}

The BER might be assessed utilizing stochastic (Monte Carlo) PC recreations. On the off chance that a basic transmission channel model and information source model is expected, the BER may likewise be ascertained systematically. A case of such an information source model is the Bernoulli source. Case of straightforward diverts models utilized as a part of data hypothesis is:

- Binary symmetric channel (utilized as a part of examination of unraveling blunder likelihood if there should be an occurrence of non-burst bit mistakes on the transmission channel)

- Additive white Gaussian commotion (AWGN) channel without blurring.

A most dire outcome imaginable is a totally irregular channel, where commotion thoroughly commands over the valuable sign. These outcomes in a transmission BER of half (gave that a Bernoulli double information source and a twofold symmetrical channel are expected, see beneath) BER examination amongst BPSK and differentially encoded BPSK with dim coding working in background noise. In a boisterous channel, the BER is frequently communicated as a component of the standardized transporter to-clamor proportion measure signified Eb/N0, (vitality per bit to commotion power unearthly thickness proportion), or Es/N0 (vitality per adjustment image to clamor phantom thickness). For instance, on account of QPSK adjustment and AWGN channel, the BER as capacity of the Eb/N0 is given by: Individuals as a rule plot the BER bends to portray the execution of a computerized correspondence framework. In optical correspondence, BER (dB) versus Gotten Power $(\mathrm{dBm})$ is generally utilized; while in remote correspondence, BER (dB) versus SNR ( $\mathrm{dB}$ ) is utilized. Measuring the bit blunder proportion people groups pick the proper forward mistake redresses codes. Since most such codes adjust just piece flips, yet not bit-insertions or bit-cancellations, the Hamming separation metric is the suitable approach to gauge the quantity of bit blunders. Numerous FEC coders likewise ceaselessly measure the current BER. A more broad method for measuring the quantity of bit mistakes is the Levenshtein separation. The Levenshtein separation estimation is more proper for measuring crude channel execution before casing synchronization, and when utilizing mistake redress codes intended to right piece insertions and bit-cancellations.

Published By:

Blue Eyes Intelligence Engineering 


\section{SIMULATION PARAMETERS:}

\begin{tabular}{|c|c|}
\hline variables & Values \\
\hline Technique & 16Qpsk \\
\hline Bandwidth & $20 \mathrm{MHZ}$ \\
\hline No of subcarriers & 64 \\
\hline Code length & 16 \\
\hline Fast fourier transform & 256 \\
\hline Channel used & AWGN \\
\hline
\end{tabular}

\section{SIMULATION RESULTS:}

Results evaluated under various schemes specifically BPSK \& 16-Phase shifty keying with FFT size two fifty six .It is tested under two fifty six fast fourier transform with binary phase shift keying technique also 16 phase shifty keying technique .

The bit error rate been calculated with various values so that bit error rate for binary phase shifty keying system is $10^{\wedge}-5$ and BER for 16 psk system. MIMO OFDM is maintain good values compared to binary phase shifty keying technique.

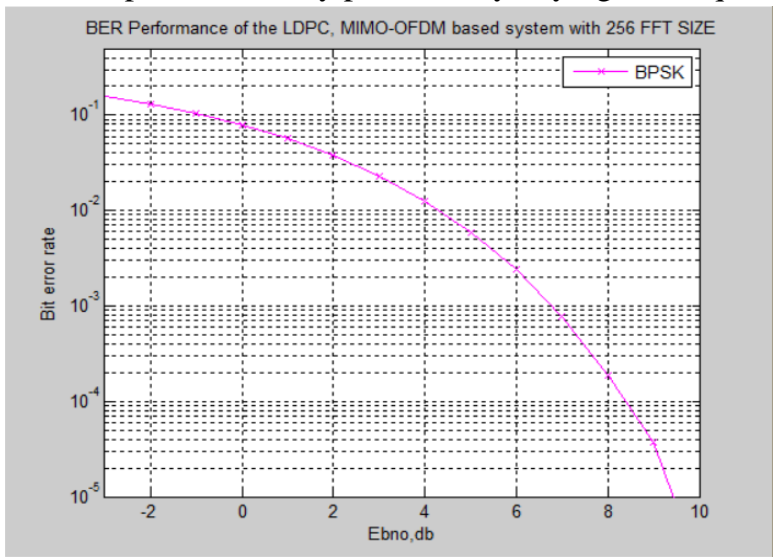

Fig.1.bit error rate with 256 fast fourier transform technique

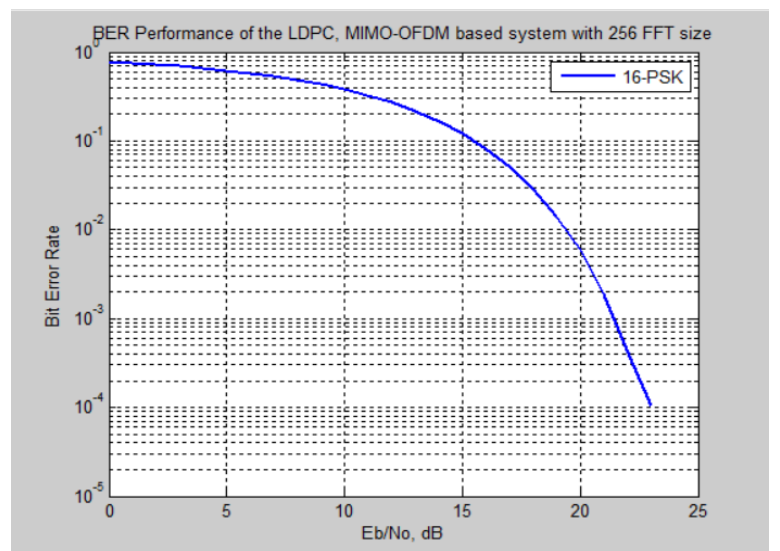

Fig.2.bit error rate using 16 psk system with 256 bits length

\section{CONCLSION:}

Multi input multi output orthogonal frequency division multiplexing system is evaluated and its performance is found very good. According to modulation techniques are varied . so our approach is far better compared to other approaches

\section{REFERENCES}

1. Priyanka Sharma and Rajesh Mehra," True single phase clocking based flip-flop design using different foundries"," International Journal of Advances in Engineering \& Technology, May, 2014. (CIJAET

2. K.Ghorbani and R. B waterhouse, "Dual polarized wide-band aperture stacked patch antenna," IEEE Trans. Antennas propagate., vol. 52, no. 8, pp.2171-2172, August 2008.

3. K. S. Ryu and A. A. Kishk, "Wideband dual polarization microstrip patch excited by hook shaped probes, "IEEE Trans Antennas propagate, vol. 56, nov 2007.

\section{ABOUT AUTHORS}

NAVABHARAT REDDY: Presently his working as a Assistant Professor in vignan institute of Technology and science at Deshmuki HYDERBAD he did his masters in communication engineering at VIT Vellore his having Industrial experience in Analog layout he also know the physical design in VLSI his research intrersts in VLSI and communications

Kiran Kumar: Presently his working as a Assistant Professor in vignan institute of Technology and science he did his masters in Embedded system engineering at VIT Chennai his having industrial experience in Embedded systems his research intrests in Embedded as well as communications

Sruti Setlam : Presently she is working has a Software developer in MNC she is having Industrial experience she completed her post graduation in communication engineering at VIT Vellore she is having vast knowledge in communication as well as Antennas she has interested in research she also worked has Intern in ISRO.

Harshitha Sirikonda : Presently she is working has a Assistant professor in Siddhartha Institute of engineering Techonlogy she completed her post graduation in communication engineering at VIT Vellore her research intrests in communication. 